\title{
DENDROLOGIA Y PROPAGACION VEGETATIVA DE Acacia horrida ("HUARANGUILLO") MEDIANTE ESTACAS INDUCIDAS EN TRES SUSTANCIAS ENRAIZANTES, USANDO TRES SUSTRATOS \\ Romiris Beatriz Pérez Contreras ${ }^{1}$, Carlos Reynel Rodríguez ${ }^{2}$ y María Manta Nolasco ${ }^{3}$.
}

\section{Resumen}

La presente investigación caracterizó dendrológicamente a la especie Acacia horrida y evaluó el mayor prendimiento de estacas para esta especie. En la propagación vegetativa se empleó tres enraizantes: miel de abeja, agua de coco y ácido indol butírico (AIB), a tres concentraciones diferentes cada una, empleando tres sustratos diferentes conformados por humus, tierra agrícola y arena. El material experimental proviene del distrito de San José, provincia de Chincha, departamento de Ica, Perú. La caracterización dendrológica se llevó a cabo en el Herbario MOL de la Facultad de Ciencias Forestales y la propagación vegetativa, en las instalaciones del Vivero Forestal de la Universidad Nacional Agraria La Molina (UNALM), entre los meses de Abril y Agosto de 1999.

Se confirmó la identidad de la especie dentro del género de las Acacias, confrontando material botánico con otras especies que causaban duda taxonómica, tales como Acacia macracantha y Acacia huarango. Asimismo, los mejores resultados en cuanto al prendimiento de las estacas (callosidad y enraizamiento), se observaron en el sustrato 2 (humus, tierra agrícola y arena en la proporción 4:1:1 respectivamente).

Palabras claves: dendrología, silvicultura, huaranguillo, Acacia horrida, Acacia macracantha, Acacia huarango.

Abstract

In this research the species Acacia horrida was dendrologically characterized and an increased number of cuttings which took root were evaluated. During the vegetative propagation three reproductive substances were used: honey, coconut water and indol butiric acid (IBA), and they were added to three different types of substrates made up of humus, agricultural soil and sand. The material for the dendrologic characterization and for the reproductive propagation came from San José district, province of Chincha in Ica, Peru. The dendrologic description was done at the MOL herbarium of the Forestry Faculty, and the vegetative propagation at the Forestry nursery of La Molina Agrarian University, between April and August 1999.

The species was confirmed to be comprised in the Acacia genus, this was fulfilled by comparing the botanic material with that from other species which caused taxonomic doubt, as Acacia macracantha and Acacia huarango. The best results in the taking root (to be well grounded and the callosity development) of cuttings were observed when coconut water $(100 \%)$ was used and by using the substratum number two (humus, agricultural soil and sand in the ratio 4:1:1).

Keywords: dendrology, silviculture, huaranguillo, Acacia horrida, Acacia macracantha, Acacia huarango.

\section{Introducción}

Desde hace varios años el "Huaranguillo" (Acacia horrida) está adquiriendo creciente importancia en el Perú. Esta especie por ser leguminosa, tiene una función restauradora y enriquecedora de la fertilidad natural de los suelos, favoreciendo la calidad de los ambientes asociados a ella. En la actualidad no se cuenta con información dendrológica y silvicultural precisa para el Perú, pese a que se viene propagando en forma local y se encuentra difundida a lo largo de toda la costa peruana.

Acacia horrida tiene múltiples usos, sin embargo el uso principal es de cortinas rompevientos y cercos vivos asociados a cultivos agrícolas. Los parceleros locales sugieren que ésta forma de uso, logra disminuir la velocidad del viento y reduce la evapotranspiración. Por lo tanto eleva la humedad del suelo y reduce el efecto erosivo del viento, en los

\footnotetext{
${ }^{1}$ Facultad de Ciencias Forestales. Universidad Nacional Agraria La Molina. Apartado postal 456 Lima 100.

${ }^{2}$ Docente de la Facultad de Ciencias Forestales, Universidad Nacional Agraria La Molina. Apartado postal 456 Lima 100. Correo electrónico reynel@lamolina.edu.pe

${ }^{3}$ Docente de la Facultad de Ciencias Forestales, Universidad Nacional Agraria La Molina. Apartado postal 456

Lima 100. Correo eletrónico mmanta@lamolina.edu.pe
} 
campos de cultivo contribuyendo a mejorar la productividad del valle. Adicionalmente presenta un alto potencial en apoyo de actividades pecuarias, especialmente en la crianza del ganado caprino (como forraje), en apicultura y curtiembre.

La presente investigación tiene como objetivos el ampliar los conocimientos sobre la dendrología y la propagación vegetativa de Acacia horrida a través de la determinación del mejor tratamiento basándose en enraizantes y el tipo de sustrato adecuado para el mayor prendimiento en estacas de esta especie.

\section{Revisión de literatura}

Cialdella (1984) y Rossl (s.f.), mencionan que el género Acacia posee aproximadamente 900 especies distribuídas en las regiones tropicales y subtropicales de América, Africa, Asia y Australia; aproximadamente 40 de ellas se encuentran representadas en nuestro país y distribuidas mayormente en provincias de la costa y sierra peruana.

El "Huaranguillo" Acacia horrida (L) Willd, es un árbol introducido proveniente del sur de Africa. En el Perú se encuentra bien adaptado y aparentemente en proceso de naturalización en gran parte de la costa, (desde Piura hasta Tacna), principalmente en la costa central; en el litoral en zonas cercanas al mar, en suelos arenosos salados, con fuerte brisa marina, como por ejemplo en el área del balneario Santa Rosa. En la sierra se le observa en los valles interandinos como Huánuco y en la ceja de montaña en Tingo María y Oxapampa desde los 800 a 2000 m.s.n.m.

En el Perú, no se tiene información sobre estudios preliminares sobre la propagación de esta especie. Existen sin embargo, información sobre otras especies forestales adaptadas a la misma zona donde se adapta Acacia horrida. Vilela (1990) evaluó el comportamiento de tres activadores naturales en el brotamiento y enraizamiento de estacas de "Algarrobo" Prosopis sp ( Mimosoideae), encontrando que los máximos porcentajes de enraizamiento (54\%) alcanza los 49 días, bajo un tratamiento de $56 \mathrm{ml}$ de miel de abeja.

La información preexistente sugiere que el empleo de diferentes sustratos o estimulantes de enraizamiento, permiten incrementar los porcentajes de prendimiento de estacas. De este modo se favorece la propagación de especies forestales en las zonas costeras del Perú, aumentando el número de especies alternativas para la reforestación.

\section{Materiales y métodos}

Estudio Dendrológico

El estudio dendrológico consistió en el marcado y monitoreo de individuos a lo largo de un año, para conseguir muestras botánicas completas. Estas fueron obtenidas por procedimientos estándares de colección (Ríos, 1982), y fueron acondicionadas y depositadas en el Herbario de la Facultad de Ciencias Forestales de la Universidad Nacional Agraria La Molina (MOL).

Las muestras fueron confrontadas con las colecciones de referencia del Herbario MOL y se identificó a la especie, consultando con la bibliografía especializada, mediante un estudio morfológico comparativo. Posteriormente, los especímenes fueron remitidos al Herbario del Royal Botanic Gardens, Kew, Londres (K), para confirmar su determinación .

Se preparó una descripción detallada de la planta incluyendo sus caracteres de campo.

Estudio Silvicultural

El presente estudio se realizó en el Vivero Forestal de la Universidad Nacional Agraria La Molina, ubicado en Viñas Bajas, distrito de La Molina, provincia y departamento de Lima. El vivero está ubicado a $12^{\circ} 05^{\prime} \mathrm{S}$ y $76^{\circ} 57^{\prime} \mathrm{W}$ a 243.7 m.s.n.m. con una precipitación de $18.3 \mathrm{~mm}$, en una zona de vida desierto desecado Sub - tropical (dd-S) según Holdridge (1960).

Los suelos de la zona son franco arenosos.

Se emplearon los materiales y equipos convencionales para la propagación vegetativa por estacas.

El material genético para la realización del experimento, consistió en 950 unidades de estacas del nivel medio de la copa del "Huaranguillo", obtenidas de un total de 10 árboles de buena conformación vegetativa, que se encontraban formando pequeños bosquetes en la zona de San José, distrito de Chincha Alta, provincia de Chincha, departamento de Ica (aproximadamente a $13^{\circ} 26^{\prime} \mathrm{S}$ y a $76^{\circ} 17^{\prime} \mathrm{W}$ ), a 298 m.s.n.m., donde la precipitación promedio anual es $11.5 \mathrm{~mm}$, y corresponde a la zona de vida desierto Pre - Montano (d-PM) según Holdridge (1960). Las estacas fueron recolectadas entre el 9 y 10 de abril de 1999. Estas tuvieron $30 \mathrm{~cm}$ de longitud, con tres yemas en promedio, diámetro que variaba entre 1 a 2 $\mathrm{cm}$ y sin hojas (cortándolas cuando fue necesario).

Los sustratos empleados estaban conformados por humus, tierra agrícola y arena en las siguientes proporciones respectivamente:

Sustrato 1: 1,1,4

Sustrato $2: 4,1,1$

Sustrato $3: 2,2,2$

Finalmente el material químico utilizado en la propagación vegetativa de estacas, consistió en tres enraizantes (reguladores de crecimiento):

TA: miel de abeja $(5,10,15 \mathrm{cc} / \mathrm{l})$,

TB: agua de coco $(25,50,100 \%) \mathrm{y}$

TC: AIB (50, 100, $150 \mathrm{ppm})$.

Los resultados de estos tratamientos fueron confrontados con el Testigo (al cual no se le aplicó enraizantes, sin embargo se remojaron 100 estacas en agua por 24 horas y se utilizó arena como sustrato).

El ensayo se realizó en una cama de $1 \times 10 \mathrm{~m}$ con una profundidad de $20 \mathrm{~cm}$, la cual se dividió de la siguiente manera: para la parcela Testigo una 
dimensión de 1x1m, para los tres sustratos correspondientes se dividieron tres parcelas de $1 \times 3 \mathrm{~m}$ cada una, distribuyéndolas aleatoriamente, luego se los etiquetó para su posterior evaluación. La cama a ensayar se encontraba orientada de Este a Oeste. Los sustratos fueron deshierbados, nivelados y finalmente desinfectados, listos para la instalación del experimento (estacado).

Los enraizantes fueron aplicados mediante el método de remojo prolongado a $10 \mathrm{~cm}$ de la base de las estacas, para esto se separó 9 grupos con 90 estacas cada uno. Para cada tipo de enraizante se utilizó 3 grupos de 90 estacas cada una para las tres concentraciones que le correspondía a cada enraizante. El tiempo utilizado en el remojo fue de 12 horas para los enraizantes naturales: miel de abeja y agua de coco, y para el enraizante sintético AIB el tiempo fue de 3 horas.

Para la instalación del experimento se siguió el diseño de los tratamientos establecidos previamente, se humedecieron los sustratos y se procedió a estacar directamente a una profundidad de $10 \mathrm{~cm}$ y un espacio entre estacas de $10 \mathrm{~cm}$.

Las labores culturales realizadas fueron: riego aplicado cuatro veces por semana durante los dos primeros meses, y luego de acuerdo al requerimiento del ensayo, y el deshierbo que se realizó una vez por semana.

Se empleó el tinglado sobre la cama, para evitar la incidencia directa de los rayos solares.

El diseño estadístico empleado para el ensayo es el Experimento Factorial en un Diseño de Bloques Completo al Azar.

El seguimiento del experimento se realizó por un tiempo de 4 meses y 1 semana a partir del 12 de Abril hasta el 20 de agosto de 1999.

Para la evaluación de los resultados en la propagación vegetativa se cuantificó en primer lugar el prendimiento de las estacas de "Huaranguillo", através de de callos, raíces desarrolladas y callos en estado de latencia.

\section{Resultados y discusión}

Dendrología

La identidad del "Huaranguillo" corresponde a Acacia horrida, aclararándose las diferencias entre esta especie y las dos especies nativas que habitan en la misma área del estudio: Acacia macracantha y Acacia huarango.

\section{Propagación Vegetativa}

El material químico que presento el mayor número de estacas prendidas (185) fue el obtenido con el agua de coco; así mismo el sustrato que permitió obtener un mayor número de estacas prendidas fue el sustrato 2 (4partes de humus, una parte de tierra agrícola y una de arena), tal como se muestra en la Tabla 1 y 2.

En el Testigo sólo cinco (5) estacas formaron callosidad, pero ninguna logró enraizar, lo que demuestra que la especie requiere sustancias inductoras de enraizamiento para su prendimiento (Tabla 1).

Tabla 1. Número de estacas prendidas durante los cuatro meses

\begin{tabular}{|c|c|c|c|c|c|c|c|c|c|c|}
\hline SUSTRATO & TA1 & TA2 & TA3 & TB1 & TB2 & TB3 & TC1 & $\mathrm{TC} 2$ & TC3 & TOTAL \\
\hline 1 & 14 & 16 & 19 & 19 & 19 & 20 & 16 & 17 & 17 & 157 \\
\hline 2 & 15 & 16 & 20 & 21 & 22 & 22 & 17 & 19 & 24 & 176 \\
\hline 3 & 14 & 14 & 18 & 20 & 20 & 22 & 17 & 18 & 23 & 166 \\
\hline Total/Concent & 43 & 46 & 57 & 60 & 61 & 64 & 50 & 54 & 64 & 499 \\
\hline Total /Tratam & & 146 & & & 185 & & & 168 & & 499 \\
\hline TESTIGO & & & & & & & & & & 5 \\
\hline \begin{tabular}{|l|} 
Gran TOTAL \\
\end{tabular} & & & & & & & & & & 504 \\
\hline
\end{tabular}

Tabla 2. Resultado ordenado de números de estacas prendidas durante los cuatro meses $\sqrt{ } \mathrm{X}+1$

\begin{tabular}{|l|l|l|l|l|l|l|l|l|l|r|}
\hline \multirow{2}{*}{ SUSTRATO } & \multicolumn{1}{|c|}{ TRATAMIENTO } \\
\cline { 2 - 12 } & TA1 & TA2 & TA3 & TB1 & TB2 & TB3 & TC1 & TC2 & TC3 & TOTAL \\
\hline 1 & 3.87 & 4.12 & 4.47 & 4.47 & 4.47 & 4.58 & 4.12 & 4.24 & 4.24 & 38.58 \\
\hline 2 & 4.00 & 4.12 & 4.58 & 4.69 & 4.80 & 4.80 & 4.24 & 4.47 & 5.00 & 40.70 \\
\hline 3 & 3.87 & 3.87 & 4.36 & 4.58 & 4.58 & 4.80 & 4.24 & 4.36 & 4.90 & 39.56 \\
$\begin{array}{l}\text { Total/concentr } \\
\text { aciones }\end{array}$ & 11.74 & 12.11 & 13.41 & 13.74 & 13.85 & 14.18 & 12.60 & 13.07 & 14.14 & 118.84 \\
\hline Total de trata. & \multicolumn{7}{|c|}{37.26} & \multicolumn{7}{|c|}{41.77} & \multicolumn{3}{|c|}{39.81} & 118.84 \\
\hline PROMEDIO & 3.91 & 4.04 & 4.47 & 4.58 & 4.62 & 4.73 & 4.20 & 4.36 & 4.71 & 4.40
\end{tabular}

Tabla 3. Análisis de variancia.

\begin{tabular}{|l|l|l|l|l|l|l|l|}
\hline F.V. & G.L. & S.C. & C.M. & F. calc. & $\begin{array}{l}\mathrm{F}_{(2.16)} \text { tab. } \\
\forall=0.01\end{array}$ & $\begin{array}{l}\mathrm{F}_{(2.16)} \text { tab. } \\
\forall=0.05\end{array}$ & \\
\hline Bloques & 2 & 84.8518 & 42.430 & 20.90 & 6.23 & 3.63 & $* *$ \\
Sustrato (sust.) & 2 & 20.0740 & 10.035 & 4.94 & 6.23 & 3.63 & $*$ \\
Conc. Tratamientos & 2 & 61.6296 & 30.815 & 15.18 & 6.23 & 3.63 & $* *$ \\
Sust. Por Conc. Trat. & 4 & 5.7038 & 1.426 & 0.70 & 4.77 & 3.01 & n.s. \\
Error & 16 & 32.4851 & 2.0303 & - & & & \\
Total & 26 & 204.7443 & - & - & & & \\
\hline
\end{tabular}

En la Tabla 3 se muestra que hay diferencia altamente significativa (nivel del 1\%) entre las sustancias enraizantes y entre las concentraciones de dichos reguladores. Respecto a los sustratos 1, 2 y 3 utilizados en el experimento, encontramos que difieren significativamente con un nivel del 5\% (Tabla 3).

Los resultados obtenidos muestran que no existen suficientes evidencias estadísticas para demostrar interacción entre las concentraciones de las sustancias enraizantes y los sustratos (Tabla 3). 


\begin{tabular}{|c|c|c|c|}
\hline \multicolumn{4}{|c|}{$\begin{array}{l}\text { Tabla 4. Resultados de la concentración de los } \\
\text { tratamientos ordenados para la prueba de Tuckey }\end{array}$} \\
\hline $\begin{array}{l}\mathrm{N}^{\circ} \text { Concentraciones } \\
\text { Tratamientos }\end{array}$ & \begin{tabular}{|l|} 
Orden de \\
Mérito
\end{tabular} & Rendimiento & $\begin{array}{l}\text { Prueba de Tuckey } \\
\forall=0.05\end{array}$ \\
\hline TB & 1 & 20.556 & 3.65 \\
\hline $\mathrm{TC}$ & 2 & 17.889 & \\
\hline TA & 3 & 17.000 & \\
\hline Sy $=0.475$ & \multicolumn{3}{|c|}{$\overline{\mathrm{T} \mathrm{B}}-\overline{\mathrm{TC}}=$} \\
\hline $\operatorname{AES}(\mathrm{T}) 0.05=3.65$ & \multicolumn{3}{|l|}{$\overline{\mathrm{TB}}-\overline{\mathrm{T} A}=$} \\
\hline $\operatorname{ALS}(\mathrm{T}) 0.05=1.73$ & \multicolumn{3}{|c|}{$38 \overline{\mathrm{TC}}-\overline{\mathrm{TA}}=$} \\
\hline
\end{tabular}

Tabla 5. Resultados de sustratos ordenados para la prueba Tuckey

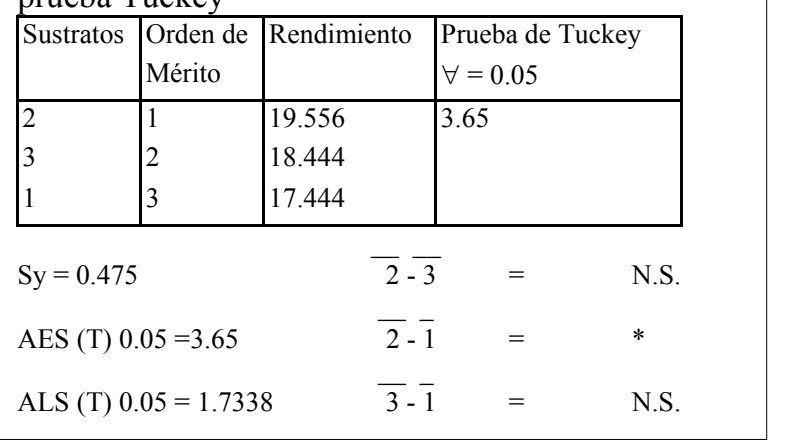

En la Tabla 4 se muestra la efectividad de las sustancia enraizantes, donde el agua de coco (TB3) obtuvo 184 estacas prendidas, resultando ser el mejor de acuerdo a la prueba de comparación múltiple de Tuckey a un nivel de significancia del 5\%.

Luego de la prueba de comparación múltiple de Tuckey (Tabla 5) para los sustratos, se demostró que para un nivel de significación del $5 \%$, el sustrato 2 (4:1:1) obtuvo el mayor número de estacas prendidas (176) bajo cualquier regulador de crecimiento.

\section{Conclusiones}

La especie forestal comunmente llamada "Huaranguillo" en la costa central del Perú es Acacia horrida (L) Willd, y se encuentra en proceso de naturalización en el Perú.

El enraizante que produjo el mayor número promedio de estacas prendidas fue el agua de coco, seguido por el AIB y por último la miel de abeja. Así mismo, a mayor concentración de los enraizantes, mayor número de estacas prendidas.

El mejor resultado promedio para el prendimiento de las estacas de esta especie se produjo en el sustrato 2 (4:1:1), seguido por el sustrato $3(2: 2: 2)$ y finalmente por el sustrato 1 (1:1:4).
Los enraizantes (reguladores de crecimiento) influyen en el prendimiento de las estacas de Acacia horrida en los tres sustratos. Asi mismo, el tipo de sustrato influye en el prendimiento de las estacas de la especie.

El agua de coco es un enraizante que tiene la ventaja de ser un producto económico y de fácil uso para el productor.

\section{Recomendaciones}

Debido al lento crecimiento de las plántulas provenientes de la reproducción sexual, y a la destrucción de la mayor parte de las semillas debido al ataque de insectos del orden coleoptera, es preferible utilizar la propagación vegetativa, en esta especie, ya que se obtienen plantas más precoces que entran en producción en menor tiempo.

Ampliar los estudios en propagación vegetativa de Acacia horrida, empleando mayores concentraciones de miel de abeja.

Ampliar el tiempo de evaluación en el sustrato 2 (4:1:1) con la finalidad de comprobar si hay un mayor número y desarrollo de raíces.

Reducir la longitud de las estacas en $5 \mathrm{~cm}$, ya que la temperatura del aire en el vivero se elevó, tendiendo a estimular el desarrollo de las yemas con anticipación al de las raíces, siendo importante que se logre el desarrollo de las raíces antes que las del tallo

Ensayar la propagación vegetativa de Acacia horrida, utilizando agua de coco y plantando estacas en terreno definitivo. Asi mismo ensayar la propagación vegetativa de la especie en platabandas y utilizando bolsas usando el sustrato 2 y agua de coco al $100 \%$.

Propagar vegetativamente esta especie con agua de coco al $100 \%$, después de la fructificación y antes de la floración del arbol madre, es decir entre los meses de Julio a Diciembre.

\section{Literatura citada}

Cialdella, A. 1 984. El Género Acacia (leguminosae) en la Argentina. Buenos Aires. Argentina.

Holdridge, L. 1 960. Zonas de vida natural en el Perú. Memoria explicativa sobre el Mapa Ecológico del Perú. Instituto Interamericano de Ciencias Agrícolas de la OEA. Zona Andina.

Ríos T, J. 1 982. Prácticas de dendrología tropical. Facultad de Ciencias Forestales. Lima. Perú. REDINFOR. Universidad Nacional Agraria La Molina.

Rossl, E. s.f. Relación de las plantas que se pueden sembrar en las urbanizaciones de las diferentes zonas del Perú. Lima. Perú. PRONAMACHCS.

Vilela, P. 1 990. Investigación Forestal en las zonas áridas del noreste del Perú. Piura. Perú. Instituto AgroIndustrial. 